\title{
Possible Role of Fish as Transport Hosts for Dracunculus spp. Larvae
}

\section{Christopher A. Cleveland, Mark L. Eberhard, Alec T. Thompson, Stephen J. Smith, Hubert Zirimwabagabo, Robert Bringolf, Michael J. Yabsley}

To inform Dracunculus medinensis (Guinea worm) eradication efforts, we evaluated the role of fish as transport hosts for Dracunculus worms. Ferrets fed fish that had ingested infected copepods became infected, highlighting the importance of recommendations to cook fish, bury entrails, and prevent dogs from consuming raw fish and entrails.

$\mathrm{T}$ he campaign to eradicate Dracunculus medinensis infection (Guinea worm disease) has helped 17 of 21 countries interrupt transmission (1). Endemic transmission of Guinea worm disease typically occurred via contamination of drinking water sources, resulting in community disease outbreaks. The absence of outbreaks of Guinea worm disease in Chad, coupled with increasing infections among domestic dogs in the transmission cycle (2-6), led to the hypothesis that transmission was occurring by different means.

Previous work on Dracunculus and related spirurids indicates that paratenic hosts might be used to facilitate transmission $(7,8)$. Recently, an experimental study showed $D$. medinensis worms could use tadpoles as paratenic hosts, and a naturally infected frog was detected in Chad $(9,10)$. Few data exist on the potential role of fish as paratenic hosts; however, fish are suspected on the basis of epidemiologic data in Chad (2). Previous experimental attempts to infect Nile tilapia (Oreochromis niloticus) and fathead minnows (Pimephales promelas) with D. medinensis worms were not successful (9). However, in trials with $D$. insignis worms, 2 of 7 fish species exposed to high numbers of larvae became infected with low numbers of larvae (11). Collectively, these data suggest that fish are generally resistant to infection, need to ingest very high numbers of larvae to establish infection, or have variable species susceptibility.

Alternatively, it is possible that fish might ingest infected copepods and, if consumed by a host within short Author affiliations: University of Georgia, Athens, Georgia, USA (C.A. Cleveland, S.J. Smith, R. Bringolf, M.J. Yabsley); Centers for Disease Control and Prevention, Atlanta, Georgia (M.L. Eberhard); University of Oklahoma, Norman, Oklahoma, USA

(A.T. Thompson); The Carter Center, Atlanta (H. Zirimwabagabo)

DOI: https://doi.org/10.3201/eid2309.161931 enough intervals, act as transport hosts. Our objective was to evaluate this potential role by allowing fish to ingest Dracunculus worm-infected copepods and then feed them to domestic ferrets to evaluate whether transmission could occur.

\section{The Study}

In April 2016, we used first-stage larvae (L1) from gravid D. insignis worms from raccoons (Procyon lotor) in Georgia (USA) to infect colony-reared cyclopoid copepods (7). At 21 days postinfection, $D$. insignis larvae were examined to determine if they had developed to the infective third stage (i.e., trifid tail). If $\geq 25 \%$ of copepods were infected, they were used for transmission trials. Gravid D. medinensis worms were recovered from naturally infected dogs in Chad, and L1s were used to infect cyclopoid copepods collected from N'Djamena.

We then exposed groups of 5 Nile tilapia, fathead minnows, or mosquitofish (Gambusia affinis) to groups of 50 copepods (Table). We exposed fish to copepods for 3 hours in the first day of the $D$. insignis trial; on subsequent days, we exposed fish for 2 hours. All copepods provided to fish were consumed during exposures. Individual fish were removed, euthanized by exposure to neutral buffered tricaine methane sulfonate (MS-222) followed by pithing, and dissected. We observed digested copepods and free larvae in the intestine.

We fed the euthanized fish to laboratory-raised ferrets. If fish were not immediately ingested, we mixed the fish carcasses with cat food. Ferrets were fed fish in small batches. For $D$. insignis worms, we conducted exposures using copepods infected with larvae originating from 2 female worms at 2 different times (i.e., 5 fish per day for 3 days in April 2016 and another 5 fish per day for 3 days in July 2016, resulting in exposure to $\approx 300$ copepods) (Table). Because there was only $1 D$. medinensis worm, fewer D. medinensis worm-infected copepods were available. We exposed only 1 species of fish (mosquitofish). A ferret was given 5 fish per day for 6 days for a total of $\approx 300$ copepods. (Table).

We maintained exposed ferrets for 77-134 days, then anesthetized and humanely euthanized them using $30 \mathrm{mg} /$ $\mathrm{kg}$ ketamine followed by sodium pentobarbital. We necropsied the ferrets and examined the recovered Dracunculus worms to determine sex and whether females were mated or gravid. All animal procedures were reviewed and 
Possible Role of Fish as Transport Hosts for Dracunculus Species

Table. Results of ferret exposure trials with 3 different fish species exposed to copepods infected with Dracunculus medinensis or $D$. insignis worms

\begin{tabular}{|c|c|c|c|c|c|c|}
\hline Fish species & $\begin{array}{l}\text { Dracunculus } \\
\text { sp. }\end{array}$ & $\begin{array}{c}\text { No. fish } \\
\text { consumed/no. } \\
\text { offered* }^{*}\end{array}$ & $\begin{array}{l}\text { Total no. } \\
\text { copepods } †\end{array}$ & $\begin{array}{c}\text { Days until } \\
\text { euthanasia of } \\
\text { ferret } \ddagger\end{array}$ & $\begin{array}{c}\text { Dracunculus } \\
\text { infection status } \\
\text { of ferret }\end{array}$ & $\begin{array}{l}\text { No. worms } \\
\text { recovered } \\
\text { and sex§ }\end{array}$ \\
\hline (Gambusia affinis) & $\begin{array}{l}\text { D. insignis } \\
D . \text { medinensis }\end{array}$ & & & $\begin{array}{c}91 \text { and } 134 \\
77\end{array}$ & + & $\begin{array}{c}0 \\
1 \mathrm{M} / 11 \mathrm{~F}\end{array}$ \\
\hline Tilap & & & & 91 and 134 & + & \\
\hline $\begin{array}{l}\text { Fathead minnow (Pimephales } \\
\text { promelas) }\end{array}$ & $D$. & $30 / 30$ & 3 & 91 & + & $1 \mathrm{M}$ \\
\hline \multicolumn{7}{|c|}{$\begin{array}{l}\text { *In groups of } 5 \text { fish/day for } 6 \text { days. } \\
\dagger \geq 25 \% \text { of copepods infected. } \\
\ddagger \text { The } D \text {. insignis worm-exposed ferrets have } 2 \text { entries for days until euthanasia because these animals were exposed to fish at } 2 \text { different time points with } \\
\text { copepods infected with larvae from } 2 \text { different worms. } \\
\text { §All worms were recovered from the subcutaneous tissues of the limbs. } \\
\text { TOf these } 6 \text { female worms, } 5 \text { were gravid, indicating a male worm either was missed or had died before necropsy. }\end{array}$} \\
\hline
\end{tabular}

approved by the University of Georgia's Institutional Animal Care and Use Committee (no. A2014 11-010).

Of the 3 ferrets we fed fish that had ingested $D$. insignis worm-infected copepods, 2 were infected (Table). One ferret was infected with $6 \mathrm{D}$. insignis females (5 gravid), the other with 1 male worm (Table). The 1 ferret fed fish exposed to $D$. medinensis worm-infected copepods became infected with 12 worms; female worms were mated but not gravid because of their young age (Table).

\section{Conclusions}

The infection of ferrets with Dracunculus spp. worms after consuming fish that had eaten infected copepods demonstrates a novel transmission route. The unprecedented increase in the number of $D$. medinensis worm infections in dogs in Chad suggests the potential role of aquatic paratenic hosts $(2,12)$. Classically, paratenic hosts become infected and facilitate transmission by bridging a trophic level, maintaining long-term infections, or concentrating larger worm burdens in their tissues (3). We suggest that fish can serve the role of transport hosts because fish did not have disseminated Dracunculus worm infection develop in our initial trials (C.A. Cleveland, unpub. data). Because most cases of Guinea worm disease occur in areas known for intense artisanal fishing and residents' dependence on fish protein, it is likely that fish and other aquatic animals play a role in transmission.

In 2014, preventive measures such as cooking fish thoroughly, burying fish entrails, and preventing dogs from consuming fish entrails were implemented in Chad. By May 2015, interventions were implemented in $>50 \%$ of at-risk communities (1). Although limited, surveys for natural infections in fish from Chad's Chari River have not detected D. medinensis larvae (2; C.A. Cleveland, unpub. data). However, our findings suggest that the proposed intervention strategies involving fish are relevant and should continue. It is unclear what happens in Chad to small fish caught by fishermen; the fish might be consumed whole without cooking or, more likely, are discarded where dogs could consume them. During previous surveys of fish for $D$. medinensis worms, large numbers of copepods were observed in their gastrointestinal tracts, supporting their potential role as transport hosts (C.A. Cleveland, unpub. data). Despite the interventions implemented in Chad, sporadic dog and human infections are still reported, suggesting a need for continued educational campaigns.

The recent report of a natural amphibian paratenic host, combined with the results of our study, indicates that the transmission of $D$. medinensis worms is not as simple as once believed (10). Despite the highly successful eradication campaign, 4 countries (South Sudan, Mali, Ethiopia, and Chad) still report endemic D. medinensis worm transmission. All 4 countries now report infections in dogs, so novel intervention and eradication strategies are needed.

Although our study showed that fish can transmit Dracunculus larvae to ferrets, many questions remain. For example, it is likely that different fish species feed on copepods at different rates and have different gastrointestinal tract transit times. This might also explain why individual exposure of mosquitofish to $D$. medinensis or $D$. insignis worms led to infection in only the $D$. medinensis-exposed ferrets; the $D$. insignis-exposed fish were fed to ferrets an hour later than $D$. medinensis-exposed fish. It is possible that mosquitofish transit material through the gastrointestinal tract faster than the other species. Alternatively, the ferrets may not have become infected simply because, as previous work has shown, not all ferrets exposed to Dracunculus worms become infected (12). Additional data are especially needed for those fish species that might be caught and ingested by humans or dogs in Guinea worm-endemic countries. Furthermore, 2 fish species retained $D$. insignis larvae in their tissues for 7-11 days, demonstrating the need for further experimental and field work on the role of fish as paratenic hosts for Dracunculus spp. worms (6).

\section{Acknowledgments}

We thank Bob Ratajczak and Matthew Walter for assistance with this project. 
The Carter Center funded this research; a full listing of supporters is available at http://www.cartercenter.org/donate/ corporate-government-foundation-partners/index.html. Additional support was provided by the wildlife management agencies of the Southeastern Cooperative Wildlife Disease Study member states through the Federal Aid to Wildlife Restoration Act (50 Stat. 917) and by a US Department of the Interior Cooperative Agreement. Additional support for C.A.C. was provided by the ARCS Foundation (Atlanta Chapter) and the Shikar Safari Club, and for A.T. by the National Science Foundation-funded Population Biology of Infectious Diseases REU site at the University of Georgia.

Mr. Cleveland is a PhD student in the Warnell School of Forestry and Natural Resources and the Southeastern Cooperative Wildlife Disease Study at the University of Georgia. He has broad interests in parasite life cycles and transmission dynamics, wildlife management, and the ecology of infectious diseases.

\section{References}

1. Hopkins DR, Ruiz-Tiben E, Eberhard ML, Roy SL. Progress toward global eradication of dracunculiasis, January 2014-June 2015. MMWR Morb Mortal Wkly Rep. 2015;64:1161-5. http://dx.doi.org/10.15585/mmwr.mm6441a1

2. Eberhard ML, Ruiz-Tiben E, Hopkins DR, Farrell C, Toe F, Weiss A, et al. The peculiar epidemiology of dracunculiasis in Chad. Am J Trop Med Hyg. 2014;90:61-70. http://dx.doi.org/ 10.4269/ajtmh.13-0554

3. Callaway E; The Lancet Infectious Diseases. Guinea worm disease nears eradication. Lancet Infect Dis. 2016;16:131. http://dx.doi.org/10.1016/S1473-3099(16)00020-7
4. Galán-Puchades MT. Dogs and Guinea worm eradication. Lancet Infect Dis. 2016;16:770. http://dx.doi.org/10.1016/ S1473-3099(16)30080-9

5. Eberhard ML, Ruiz-Tiben E, Hopkins DR. Dogs and Guinea worm eradication. Lancet Infect Dis. 2016;16:1225-6. http://dx.doi.org/10.1016/S1473-3099(16)30380-2

6. Anderson RC. Nematode parasites of vertebrates: their development and transmission. New York: Cabi Publishing; 2000.

7. Eberhard ML, Brandt FH. The role of tadpoles and frogs as paratenic hosts in the life cycle of Dracunculus insignis (Nematoda: Dracunculoidea). J Parasitol. 1995;81:792-3. http://dx.doi.org/10.2307/3283979

8. Eberhard ML, Yabsley MJ, Zirimwabagabo H, Bishop H, Cleveland CA, Maerz JC, et al. Possible role of fish and frogs as paratenic hosts of Dracunculus medinensis, Chad. Emerg Infect Dis. 2016;22:1428-30. http://dx.doi.org/10.3201/eid2208.160043

9. Eberhard ML, Cleveland CA, Zirimwabagabo H, Yabsley MJ, Ouakou PT, Ruiz-Tiben E. Guinea worm (Dracunculus medinensis) infection in a wild-caught frog, Chad. Emerg Infect Dis. 2016;22:1961-2. http://dx.doi.org/10.3201/eid2211.161332

10. Crichton VFJ, Beverley-Burton M. Observations on the seasonal prevalence, pathology and transmission of Dracunculus insignis (Nematoda: Dracunculoidea) in the raccoon (Procyon lotor (L.) in Ontario. J Wildl Dis. 1977;13:273-80. http://dx.doi.org/10.7589/0090-3558-13.3.273

11. Eberhard ML, Ruiz-Tiben E, Wallace SV. Dracunculus insignis: experimental infection in the ferret, Mustela putorius furo. J Helminthol. 1988;62:265-70. http://dx.doi.org/10.1017/ S0022149X00011640

12. Hopkins DR, Ruiz-Tiben E, Eberhard ML, Roy SL, Weiss AJ. Progress toward global eradication of Dracunculiasis - January 2015-June 2016. MMWR Morb Mortal Wkly Rep. 2016;65:11126. http://dx.doi.org/10.15585/mmwr.mm6540a5

Address for correspondence: Christopher A. Cleveland, University of Georgia, Warnell School of Forestry and Natural Resources, 180 E Green St, Athens, GA 30602, USA; email: ccleve@uga.edu

\section{Get the content you want delivered to your inbox.}

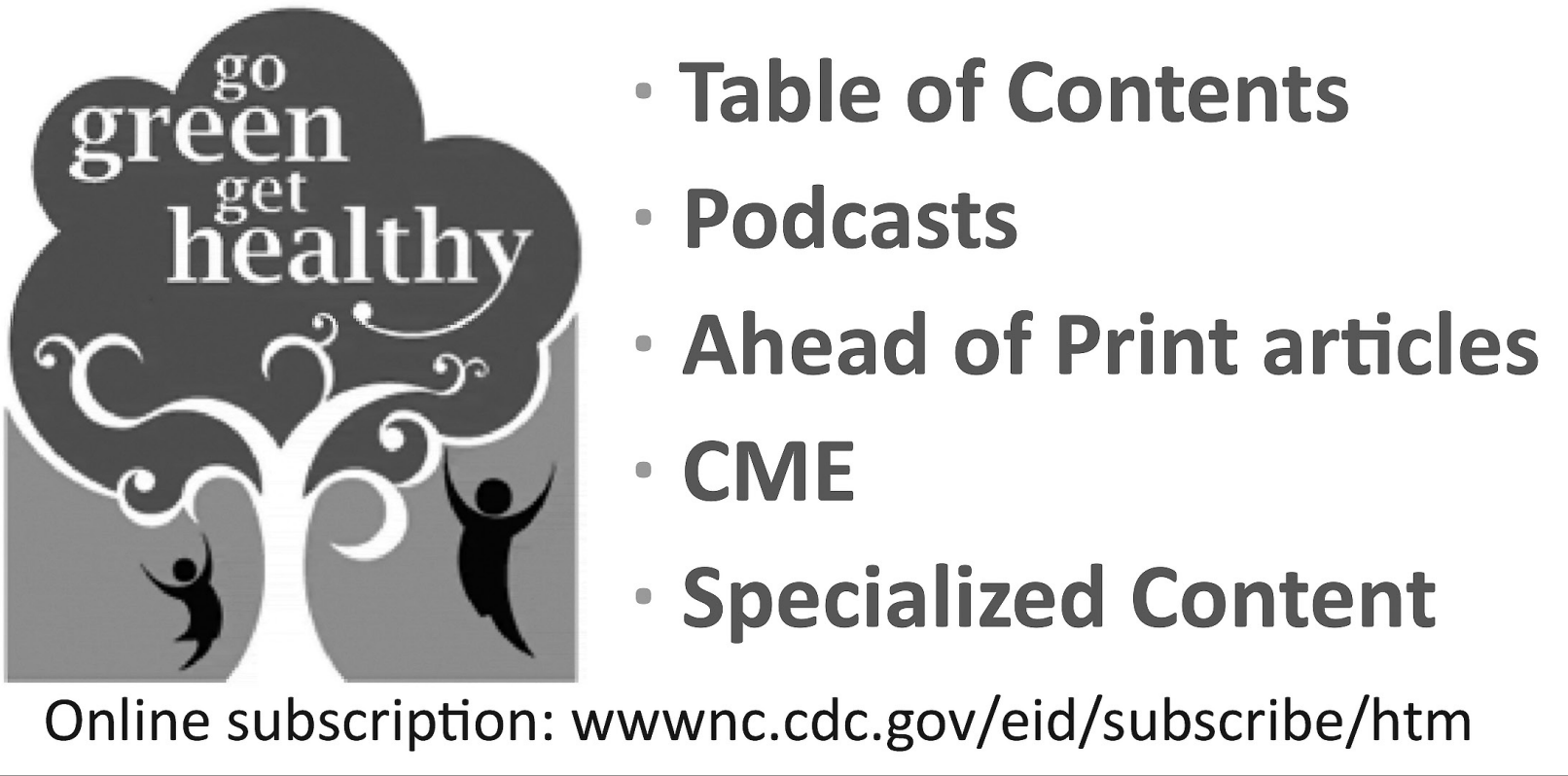

